\title{
Alternations of Septal-hippocampal System in the Adult Wistar Rat with Spatial Memory Impairments Induced by Chronic Cerebral Hypoperfusion
}

\author{
Bo-Ryoung Choi ${ }^{2}$, Kyoung Ja Kwon ${ }^{1}$, Seung Hwa Park ${ }^{3}$, Won Kyung Jeon ${ }^{4}$, \\ Seol-Heui Han ${ }^{1}$, Hahn Young Kim ${ }^{1}$ and Jung-Soo Han ${ }^{2 *}$ \\ ${ }^{1}$ Department of Neurology, Konkuk University Hospital, Center for Geriatric Neuroscience Research, IBST, Konkuk \\ University, Seoul 143-729, ${ }^{2}$ Department of Biological Sciences \& Center for Geriatric Neuroscience Research, IBST, Konkuk \\ University, Seoul 143-701, ${ }^{3}$ Department of Anatomy, College of Medicine, Konkuk University, Seoul 143-729, ${ }^{4}$ Traditional \\ Korean Medicine Converging Research Division, Korea Institute of Oriental Medicine, Daejeon 305-811, Korea
}

\begin{abstract}
In the current investigation, the status of the septo-hippocampal cholinergic pathway and hippocampal mitogen-activated protein kinase (MAPK) signaling was examined in male Wistar rats with chronic cerebral hypoperfusion, which showed cognitive deficits based on assessment on a version of the Morris water maze. Chronic cerebral hypoperfusion was induced by bilateral common artery occlusion and maintained for 12 weeks until behavioral testing. Chronic cerebral hypoperfusion was shown to induce memory impairments and microglial activation in regions of white matter, including the fimbria of hippocampus. Choline acetyltransferase expression of the basal forebrain and expression of hippocampal MAPKs was decreased in rats with BCCAo compared to control rats. The results of this study suggest that cognitive decline induced by chronic cerebral hypoperfusion could be related to dysfunction of the basal forebrain cholinergic system and reduction of hippocampal MAPK activities.
\end{abstract}

Key words: vascular dementia, neuroinflammation, memory, cholinergic, MAPK, hippocampus

\section{INTRODUCTION}

Chronic cerebral hypoperfusion is associated with the development of numerous neurological diseases (Dubois and Hebert, 2001). A moderate but persistent reduction in cerebral blood flow affects neural structure mediating memory processes over a long

Received March 16, 2011, Accepted April 12, 2011

\footnotetext{
* To whom correspondence should be addressed. TEL: 82-2-450-3293, FAX: 82-2-3436-5432 e-mail:jshan06@konkuk.ac.kr
}

period and eventually leads to the development and progression of vascular dementia (VaD) (Farkas et al., 2007), which is the second most common type of dementia following Alzheimer's disease(AD). The neurobiological markers and signaling pathway underlying AD have been well established, whereas its role in $\mathrm{VaD}$ has not been well characterized.

To understand the role of chronic cerebral hypoperfusion in the development of cognitive dysfunction in $\mathrm{VaD}$, it is important to explore the causal relationships between cerebral hypoperfusion and dependant events such as changes in neurobiological markers, signal pathway, and cognitive function. Furthermore, recognition 
of particular cellular mechanisms in the chain of events from chronic cerebral hypoperfusion to a cognitive decline may identify potential targets for effective therapies. To accomplish this, chronic cerebral hypoperfusion was induced in male Wistar rats with permanent occlusion of bilateral common carotid artery (BCCAo), which reduces the cerebral blood flow persistently as occurs in human aging, $\mathrm{AD}$ and also in $\mathrm{VaD}$ (Otori et al., 2003). Therefore, studies using animals with BCCAo have been conducted to reveal the long-term consequences of chronic cerebral hypoperfusion that leads to VaD.

One of the consistently reported characteristics in studies using animals with BCCAo is microglial activation at white matter sites (Farkas et al., 2004). The injury of white matter visualized with clinical imaging techniques has been reported to coincide with cognitive disorders in patients with VaD (Barber et al., 1999). Specifically, microglial activation labeled by OX-42 or OX-6 is much more evident in the white matter, than in the gray matter of rat brains with BCCAo (Wakita et al., 1994). Subsequently, the microglial activation may exacerbate white matter lesions, since the activated microglias produce reactive oxygen species (ROS) and proinflammatory cytokines. Thus, chronic cerebral hypoperfusion invariably induces white matter lesions and scattered foci of cortical microinfarcts either with or without hippocampal damage (Wakita et al., 1994; Ni et al., 1995). Rats with BCCAo exhibit memory deficits. For example, rats with BCCAo failed to find a hidden platform in the Morris water maze task (Ohta et al., 1997) and committed more errors in the eight-arm radial maze in comparison with control rats (Ni et al., 1994). The working memory that was measured using an object recognition task was also impaired in rats with BCCAo (Sarti et al., 2002).

Degeneration of basal forebrain cholinergic neurons (BFCN) has been found to be associated with the cognitive decline that occurs in $\mathrm{VaD}$ and $\mathrm{AD}$ because the BFCN widely innervates the hippocampus and cortex (Bartus et al., 1982; Sharp et al., 2009). It was also reported in an animal study using BCCAo that hippocampal cholinergic markers were altered in the hippocampus (Ni et al., 1995; Kumaran et al., 2008). The present experiment was conducted to examine the choline acetyltransferase (ChAT) expression and microglial inflammation of BFCN and microglial inflammation of the fimbria of hippocampus in rats with spatial memory impairments induced by BCCAo. In addition, mitogenactivated protein kinase (MAPK: ERK, JNK, p38) and MAPK phosphorylation of the hippocampus was examined in rats with BCCAo based on previous findings that dysfunction of the cholinergic septo-hippocampal system is associated with altered MAPK signaling (Williams et al., 2006; Williams et al., 2007;
Giovannini et al., 2008).

\section{MATERIALS AND METHODS}

\section{Subjects}

Thirty-five male Wistar rats were used in these experiments (12 weeks; Charles River Co., Gapeung, South Korea). The rats resided in a vivarium at Konkuk University for 2 weeks at the beginning of the experiment at a controlled temperature $\left(22 \pm 1^{\circ} \mathrm{C}\right)$ and humidity $(50 \pm 10 \%)$ on a 12 hour alternate light/ dark cycle (lights on at $0800 \mathrm{~h}$ ). Food and water were provided ad libitum throughout the experiment. All animal procedures were conducted in accordance with the approved institutional animal care procedures and guidelines of the institutional animal care and use committee of Konkuk University.

\section{Surgical procedure}

Animals were anesthetized using 5\% isoflurane and oxygen mixture and maintained with $3 \%$ isoflurane during the surgical procedure. A midline incision was made to expose the bilateral common carotid arteries, which were tightly double ligated with silk sutures. With the exception of bilateral common carotid artery occlusion, a sham-operation was conducted in which control animals were subjected to the same procedure as those conducted on the BCCAo operated animals. The rectal temperature was maintained at $37.0 \pm 0.5^{\circ} \mathrm{C}$ with a heating pad during surgery.

\section{Behavioral task}

Of the twenty-five rats that were subjected to BCCAo, three died before behavioral testing. In addition, one rat that showed large weight loss that lost their weight $80 \%$ than before the surgery was excluded from further behavior task.

As a result, ten rats per group were used for behavioral testing and neurobiological assays. Twelve weeks after the BCCAo surgery, the rats were trained in a Morris water maze task. The maze consisted of a round tank, $1.83 \mathrm{~m}$ in diameter and $0.58 \mathrm{~m}$ deep, that was filled to a depth of $35.5 \mathrm{~cm}$ with tepid $\left(26 \pm 1^{\circ} \mathrm{C}\right)$ water made opaque by the additional of non-toxic water soluble white paint (tempera). A moveable hidden circular platform (12 $\mathrm{cm}$ in diameter) was placed at a fixed location and submerged about $2 \mathrm{~cm}$ below the water surface. The maze was surrounded by white curtains on which black cloth visual stimuli of various shapes and sizes were placed. The rats were allowed to swim for a maximum 90 seconds in one trial. Every session contained five trials across two days and the total training was composed of four sessions. Each trial started from different positions in the water maze tank. A probe trial was conducted $30 \mathrm{~min}$ after the 
second session and the fourth session to assess the development of spatial bias in the maze; thus, the entire training procedure included two probe trials for each rat. During these probe trials, the rats swam with the platform retracted to the bottom of the pool for $30 \mathrm{~s}$, at which time the platform was raised to its normal position for completion of the trial. One week after the Morris water maze task with the hidden platform, all rats were tested in a Morris water maze tasks, visual platform version. The location of the visible platform varied from trial to trial in a single session of six training trials. And six rats that did not find the visual platform were considered as rats with visual problems and were excluded from behavior task analysis. A camera located above the center of the maze relayed images to a videocassette recorder and an HVS Image Analysis Computer System (Hampton, United Kingdom).

\section{Brain preparation}

Approximately 7 days after the behavioral experiments, six rats per group were used for the histology and four rats per group for western blot analysis. The rats used for the histology were deeply anesthetized with ketamin $\mathrm{HCl}(30 \mathrm{mg} / \mathrm{kg})$ and xylazine $(2.5$ $\mathrm{mg} / \mathrm{kg}$ ) cocktail, after which they were intracardially perfused with $0.01 \mathrm{M}$ phosphate buffered saline (PBS) followed by $4 \%$ paraformaldehyde (PFA). The brain was then removed and postfixed in 4\% PFA (2 d), cryoprotected in PBS containing 30\% sucrose $(48 \mathrm{~h})$, frozen on powdered dry ice and stored at $-70^{\circ} \mathrm{C}$ until processed. And the other rats used for the western blot analysis were decapitated and the brain was rapidly removed at a $4^{\circ} \mathrm{C}$ surface. The hippocampi and BFCN were dissected, rapidly frozen on powered dry ice and stored at $-80^{\circ} \mathrm{C}$ until processed.

\section{Thionin staining}

Perfused brain tissues were sectioned on microtome in $40 \mu \mathrm{m}$ coronal plane and stored in $4^{\circ} \mathrm{C}$ PBS. In each set of six serial sections, every first section was mounted on slides for thionin staining. The sections for thionin staining were mounted onto resin coated slide glass, and then dried up for 10 days. Sections were hydrated through descending concentration of ethanol and subsequently dipped in distilled water twice. Sections were stained in thionin solution for few minutes and washed in distilled water and ethanol, and then dehydrated through ascending concentration of ethanol. Then sections were defatted in Xylene and coverslipped with permount reagent.

\section{OX-6 immunohistochemistry}

Every second section in each set of six serial sections was used for immunohistochemistry (OX-6). The monoclonal antibody OX-6 (mouse anti-RT1B monoclonal antibody, BD bioscience; $1: 1,000$ ) is directed against the major histocompatibility complex (MHC) class II antigen in the microglia. For OX-6 immunoreactivity, endogenous peroxidase of free floating sections was quenched by 30 min of incubation in $3 \% \mathrm{H}_{2} \mathrm{O}_{2} / 10 \% \mathrm{MeOH}$ in PBS. The sections were then incubated for one hour at room temperature in PBS with 0.3\% Triton-X 100 containing 10\% serum (PBS-T-S). Next, the sections were then incubated with OX-6 antibody for a further $12 \mathrm{~h}$ at $4^{\circ} \mathrm{C}$ in $3 \%$ PBS-T-S solution. The sections were then incubated for $1 \mathrm{~h}$ in horse anti-mouse antibody (vector; 1 : 200) and then for $1 \mathrm{~h}$ in ExtrAvidin peroxidase conjugate (Sigma Aldrich; $1: 1,000)$. Finally, the sections were reacted with a Vector SG substrate kit (Sigma Aldrich) for peroxidase and mounted onto resin coated slides, after which they were dried for up to one week. Dried sections on slides were coverslipped with permount reagent. For quantifying the activated microglias, OX-6 antibody detected microglias were counted in corpus callosum and fimbria at six sites of brain section per an animal and statistically analysis.

\section{Western blot analysis}

Proteins for the analysis of ChAT, MAPK, and PMAPK were extracted in the following manner. Individual tissue samples were weighed and then homogenized in $5 \mathrm{vol}$ of ice-cold buffer containing $20 \mathrm{mM}$ Tris at $\mathrm{pH}$ 7.5, 5\% glycerol, $1.5 \mathrm{mM}$ EDTA, $40 \mathrm{mM} \mathrm{KCl}, 0.5 \mathrm{mM}$ dithiothreitol, and protease inhibitors (No. 539131, Calbiochem). Homogenates were centrifuged at 20,800 $\mathrm{g}$ for $30 \mathrm{~min}$ at $4^{\circ} \mathrm{C}$. The supernatant was then removed from each sample, after which an aliquot was taken for determination of the total protein concentration using Bradford Reagent. The proteins were then separated by SDS-PAGE and transferred to a PVDF membrane, which was incubated with a primary antibody (Ab) against ChAT (1:1,000, Cell Signaling), ERK (1 : 5,000, Cell Signaling), JNK ( $1: 5,000$, Cell Signaling), p38 (1 : 1,000, Cell Signaling), pERK ( $1: 3,000$, Cell Signaling), pJNK ( 1 : 3,000 , Cell Signaling), and pp38 (1:1,000, Cell Signaling). After primary incubation, blots were incubated with the horseradish peroxidase (HRP)-conjugated secondary Ab (1:2,500, Amersham Biosciences) and then visualized using an ECL system and developed using Hyperfilm (Amersham).The relative expression levels of ChAT, MAPK, and PMAPK were determined by densitometry and normalization to $\beta$-actin ( $1: 5,000$, Sigma), an invariant cytoskeletal protein.

\section{Statistic analysis}

A one-way repeated ANOVA and a t-test were conducted to assess the effects of chronic BCCAo on the spatial memory impairment, OX-6 expression, and MAPK changes. All data were expressed as the mean \pm SEM. Any p values that were less than 0.05 
were considered significant, unless otherwise specified.

\section{RESULTS}

\section{Spatial memory}

In the spatial learning with Morris water maze task, search errors that are described in detail elsewhere (Gallagher et al., 1993) were used to assess the performance accuracy of spatial learning in the water maze. During each trial, the distance of the rats from the escape platform was sample 10 times per sec, and these values were averaged in 1-s bins. The cumulative search error was then calculated as the summed 1-sec averages of this proximity measure corrected for the particular start location on each trial. As shown in Fig. 1, the sham-operated control rats quickly became proficient at locating the submerged platform during the training sessions; however, the rats with BCCAo showed poor improvements over the course of training compared with the control rats (Fig. 1). One-way repeated ANOVA showed that the between group effects (sham-operated control, BCCAo) were significant $\left(\mathrm{F}_{(1,18)}=28.13\right.$, $\mathrm{p}<0.001)$ and that the training effects (session) were significant $\left(\mathrm{F}_{(3,54)}=19.75, \mathrm{p}<0.001\right)$. There were also interaction effects between the group and training $\left(\mathrm{F}_{(3,54)}=3.23, \mathrm{p}=0.03\right)$.

In addition, although no apparent differences in performance in the first probe trial were observed, there were apparent differences in performance in the second probe trial, as assessed by the percentage of time spent in the target quadrant during the $30 \mathrm{~s}$ probe. The t-test on the second probe trial showed that the group effects were significant $(t(18)=4.01, p<0.01)$. On the first probe trial, the percentage of time spent in the target quadrant was less
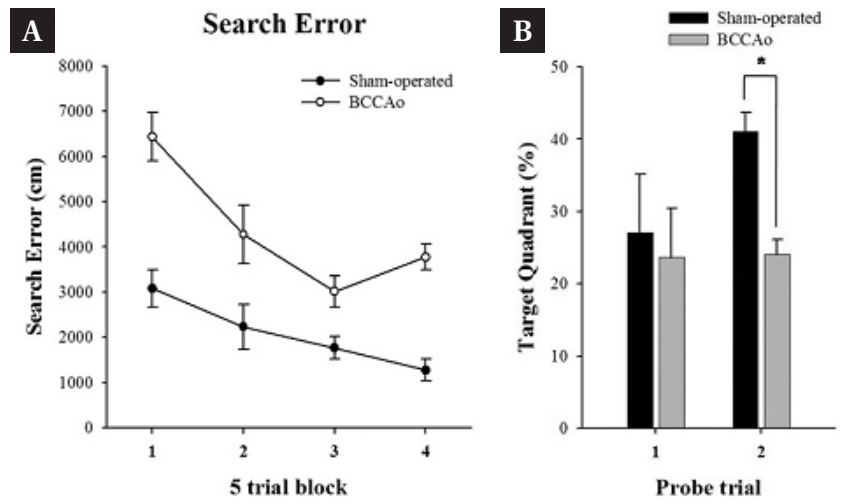

Fig. 1. Spatial memory impairments of rats with BCCAo. (A) Rats with BCCAo showed greater error when attempting to find the hidden platform compared with sham-operated rats. The value represents the mean search error of five-trial block \pm SEM (ten rats per group) (B). The percentage of time spent in the target quadrant during the $30 \mathrm{~s}$ probe. Sham-operated control rats showed the spatial bias on the second probe trial when compared with the BCCAo rats. than $30 \%$ in rats of both groups, which indicated that they did not fully learn the hidden platform location after 10 trials.

Swimming speeds during training trials and cue training: there were no significant differences in the swimming speeds between groups during training $\left(\mathrm{F}_{(1,18)}=1.03, \mathrm{~ns}\right)$. The times taken to reach the visible platform (in seconds) during the block of cue-training trials (mean \pm S.E.M.) for the two groups were as follows: shamoperated, 15.05 \pm 2.39 ; BCCAo, $(22.44 \pm 1.67)$. There were no significant differences between the sham-operated group and the BCCAo group $\left(\mathrm{F}_{(1,10)}=0.23, \mathrm{p}=0.64\right)$.

\section{ChAT reduction in BFCN and damaged in fimbria by BCCAo}

The ChAT expression of BFCN was measured in rats with chronic cerebral hypoperfusion. The ChAT expression of BFCN of rats with BCCAo was decreased by more than $30 \%$ compared with the sham-operated group $(\mathrm{t}(6)=2.43, \mathrm{p}<0.05)$ (Fig. 2A, 2B). However, upon staining for MHC class II antigen (OX-6), the OX-6 expression was not shown in the basal forebrain of rats with chronic cerebral hypoperfusion (Fig. 2C). Conversely, OX-6 expression was increased in the fimbria of hippocampus (Fig. $2 \mathrm{D}, 2 \mathrm{E}$ ), which was statistically different from the sham-operated
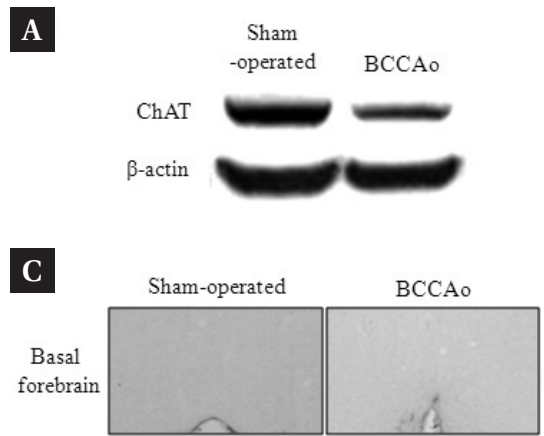

D
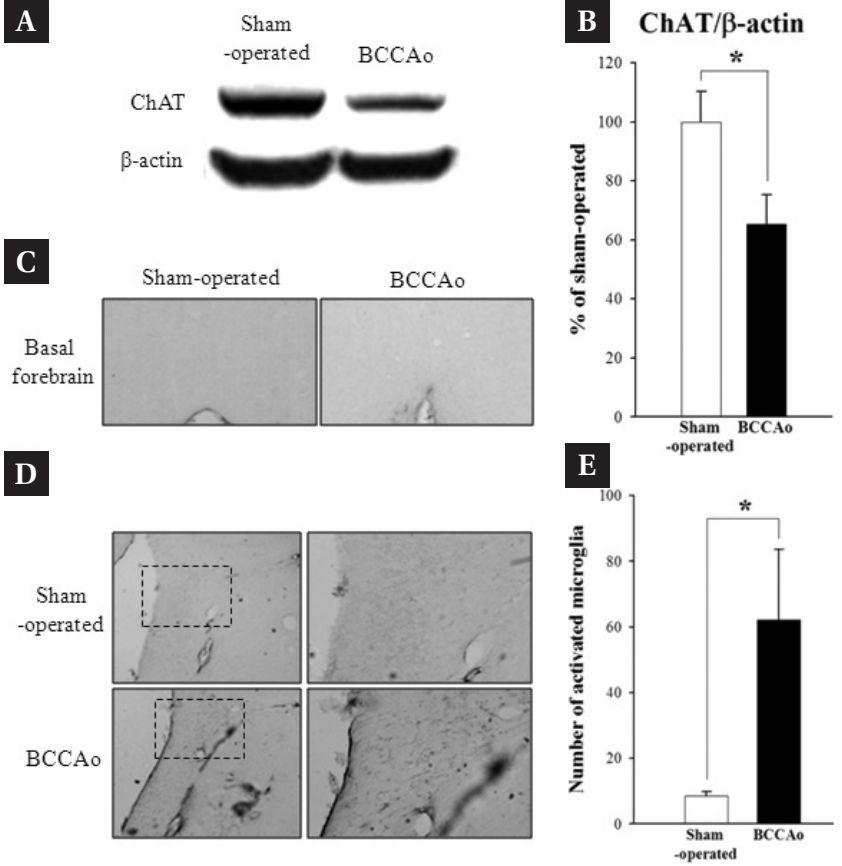

Fig. 2. Alternations of cholinergic septo-hippocampal system in rats with BCCAo. (A, B) ChAT expression in the basal forebrain was decreased by more than $30 \%$ in rats with BCCAo compared with sham-operated rats. (C) Interestingly, OX-6 immunostaining showed that no microglial activation in the basal forebrain occurred in both the sham-operated and the BCCAo group. (D, E) Microglial cells were more activated in fimbria of rats with BCCAo than in those of sham-operated rats. 
control $(\mathrm{t}(10)=2.50, \mathrm{p}<0.05)$.

\section{Hippocampal neuron and microglial activation in white matter area}

Upon thionin staining of the hippocampus, no apparent neuronal cell death was observed in rats with BCCAo, but neuronal cell death occurred in the CAl regions of the hippocampus in one animal (Fig. 3A). Consistent with earlier reports conducted using BCCAo, in the present experiment, expression of OX-6 was increased in the white matter regions such as the corpus callosum, external capsule, and internal capsule (Fig. 3B). In particular, the number of activated microglia in the corpus callosum was statistically higher

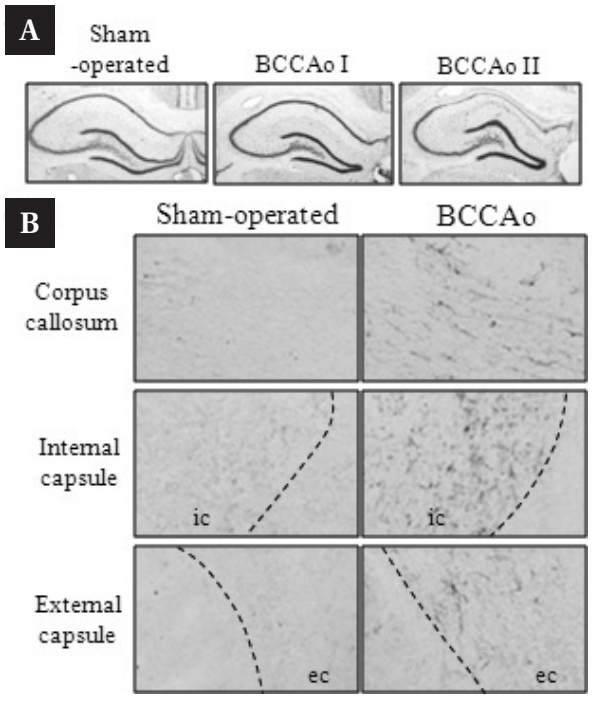

Fig. 3. Photomicrographs of the thionin stained hippocampi and the OX-6 immunostained white matter regions in rats with BCCAo. (A) Based on thionin staining, hippocampal CA1 neuronal cell death was not apparent in rats with BCCAo except for one animal. (B) In the BCCAo group, activated microglia was highly expressed in white matter regions such as the corpus callosum, external capsule, and internal capsule compared with sham-operated control rats. than those of sham-operated group $(\mathrm{t}(10)=2.55, \mathrm{p}<0.05)$.

\section{MAPK alterations in hippocampi of BCCAo}

Increases of ERK and p38 abundances of the hippocampus in rats with BCCAo were statistically significant compared with those of the sham-operated control $(\mathrm{t}(6)>-3.96, \mathrm{p}<0.01)$, while JNK did not show differences. Phosphorylation of the hippocampus MAPK was also analyzed in the BCCAo rats and the control rats. The extent of phosphorylation of hippocampus MAPK was evaluated with respect to MAPK abundances (pMAPK/MAPK). All phosphorylations of MAPKs (pERK, pJNK, and pp38) were significantly decreased in the hippocampus of rats with BCCAo in comparison with those of the sham-operated control $(\mathrm{t}(6)>2.51$, $\mathrm{p}<0.05)$ (Fig. 4).

\section{DISCUSSION}

In this experiment, chronic cerebral hypoperfusion was induced for 12 weeks by BCCAo of three month-old male Wistar rats. Previous studies reported that BCCAo led to reductions in cerebral blood flow (CBF) ranging from $30 \%$ to $40 \%$ of that before operation, and this remained at 52 64\% between seven and thirty days after operation (Tomimoto et al., 2003). Between 8 and 12 weeks after BCCAo, CBF was slightly reduced or not reduced (Ohta et al., 1997; Otori et al., 2003). The most common characteristic in rats with BCCAo is neuroinflammatory response in white matter and memory impairments (Farkas et al., 2007). The results of the present experiment showed that microglia was activated in the majority of the brain, especially in white matter regions such as the corpus callosum, internal capsule, and external capsule, and that memory impairment occurred in rats with BCCAo. The results of this experiment are consistent with those of earlier reports (Wakita et al., 2002; Takizawa et al., 2003; Farkas et al., 2004), which confirms that rats with BCCAo are a suitable

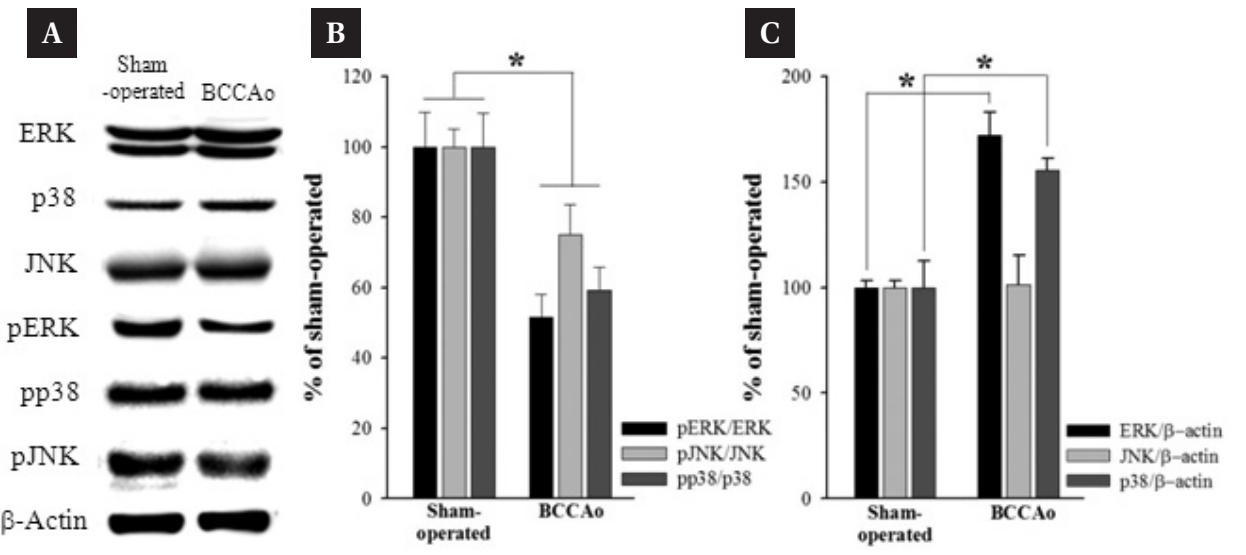

Fig. 4. Altered MAPK expression in the hippocampi of rats with BCCAo. (A) Representative western blots of MAPKs. (B) All phosphorylations of MAPKs (pERK, pJNK, and pp38) were significantly decreased in rats with BCCAo in comparison with those of sham-operated control rats. (C) ERK and p38 abundances were significantly increased in hippocampi in rats with BCCAo, while JNK showed no difference. 
animal model for chronic cerebral hypoperfusion although the CBF was not measured in this experiment.

Cognitive deficit in the BCCAo group was measured in the spatial learning version of the water maze task; however, rats with BCCAo showed comparable swimming speed to those of shamoperated control rats and were also unimpaired in the cued version of the task. The results indicate that the deficits are not attributable to general motivation or to sensorimotor deficits (Gallagher et al., 1993).

It is stated that the level of cerebral hypoperfusion by BCCAo is moderate and that the resulting neuronal loss is very regional (e.g. hippocampal CA1) (Farkas et al., 2007). It is well known that neuronal loss in the hippocampal CA1 cell layer and memory impairments occurs in the brain of rats with BCCAo (Ni et al., 1994; Bennett et al., 1998). However, it is not certain if neuronal loss occurs at long survival times after permanent BCCAo is induced by the initial phase of cerebral hypoperfusion, or if it is the outcome of a long-lasting neurodegeneration caused by the chronic phase of BCCAo or both. A series of studies have shown that the neuronal loss in the CAl became greater as the survival times was increased (Ni et al., 1994), and that the neuronal loss in CA1 appeared at four months, but not one month after permanent BCCAo. Unlike neuronal loss, the lesion of white matter was observed one months after permanent BCCAo and increased markedly four months after the operation (Ni et al., 1995). In the current experiment, after three months of survival time only one animal showed clear neuronal loss in the hippocampal CA1. There was no clear neuronal loss in the hippocampus in any of the animals. Nevertheless, it is still a possibility that these cognitive deficits might be caused by some minor neuronal loss induced by BCCAo.

However, white matter injury was apparent in all rats with BCCAo. The fimbria of the hippocampus was damaged by activated microglia similar to other white matter regions such as the corpus callosum, external capsule, and internal capsule. The fimbria of hippocampus is part of the hippocampal circuitry and consists of a major axon bundle that originates from the basal forebrain to the hippocampus. Animals with fimbria-fornix injury failed to learn the spatial learning task in the Morris water maze, but this was recovered with septal cell grafts (Nilsson et al., 1987). Dysfunction of septo-hippocampal systems was implicated in rats with BCCAo that showed changes in the central acetylcholine and choline contents and decreases of hippocampal cholinergic muscarinic receptor and ChAT expression. The ChAT and OX-6 expression of the basal forebrain was examined in rats with BCCAo. The abundances of ChAT were decreased in the basal forebrain of rats with BCCAo compared to control rats, but activated microglias labeled with OX-6 were not observed. When combined with earlier reports, white matter damage in the fimbria of hippocampus might cause a reduction of ChAT in the basal forebrain and alterations of hippocampal signaling.

Accordingly, we examined the status of MAPK signaling, which is a key regulator in neuroinflammation of AD (Haddad, 2004), although slight neuron loss was observed in the hippocampus in the BCCAo rats in the present experiment. In this experiment, ERK and $\mathrm{p} 38$ abundances of the hippocampus were increased and the MAPKs examined were less phosphorylated relative to the control rats. Interestingly, in our recent experiment, phosphorylations of hippocampal MAPKs were increased in rats with two months survival time after BCCAo (unreported observations). These results suggest that MAPKs reflect an ongoing process of inflammation in $\mathrm{VaD}$ and $\mathrm{AD}$.

The white matter damage caused by neuroinflammation is similar to the major pathological symptoms that occurs during $\mathrm{VaD}$ (Tomimoto et al., 1996). In addition, these white matter lesions in animal models with BCCAo share common features with VaD (Ueno et al., 2002; Tomimoto et al., 2003). The present study, which examined rats that survived for three months after BCCAo, is the first report that ChAT of the basal forebrain was decreased and that hippocamal MAPK signaling was altered. Interestingly, the findings that ChAT of the basal forebrain was decreased are strengthened by the clinical report that patients with $\mathrm{VaD}$ show cholinergic deficits in the brain and cerebrospinal fluid (CSF), and that it had significant benefits in cognition following treatment with donepezil, an acetylcholinesterase inhibitor (Roman et al., 2005; Wang et al., 2009). Moreover, the findings that the hippocampal signaling pathway related to MAPKs is altered in an animal model of $\mathrm{VaD}$ provide a new therapeutic target for the development of antidementia agents.

\section{ACKNOWLEDGEMENTS}

This work was supported by a Korea Research Foundation Grant funded by the Korean Government (KRF-2008-331-E00298) to Hahn Young Kim and a grant (kiom-2010-2) from the InterInstitutional Collaboration Research Program provided by the the Korea Research Council of Fundamental Science \& Technology (KRCF), Korea.

\section{REFERENCES}

1. Barber R, Scheltens P, Gholkar A, Ballard C, McKeith I, Ince P, Perry R and O'Brien J (1999) White matter lesions on magnetic resonance imaging in dementia with Lewy bodies, 
Alzheimer's disease, vascular dementia, and normal aging. J Neurol Neurosurg Psychiatry 67:66-72.

2. Bartus RT, Dean RL, 3rd, Beer B and Lippa AS (1982) The cholinergic hypothesis of geriatric memory dysfunction. Science 217:408-414.

3. Bennett SA, Tenniswood M, Chen JH, Davidson CM, Keyes MT, Fortin T and Pappas BA (1998) Chronic cerebral hypoperfusion elicits neuronal apoptosis and behavioral impairment. Neuroreport 9:161-166.

4. Dubois MF and Hebert R (2001) The incidence of vascular dementia in Canada: a comparison with Europe and East Asia. Neuroepidemiology 20:179-187.

5. Farkas E, Luiten PG and Bari F (2007) Permanent, bilateral common carotid artery occlusion in the rat: a model for chronic cerebral hypoperfusion-related neurodegenerative diseases. Brain Res Rev 54:162-180.

6. Farkas E, Donka G, de Vos RA, Mihaly A, Bari F and Luiten PG (2004) Experimental cerebral hypoperfusion induces white matter injury and microglial activation in the rat brain. Acta Neuropathol 108:57-64.

7. Gallagher M, Burwell R and Burchinal M (1993) Severity of spatial learning impairment in aging: development of a learning index for performance in the Morris water maze. Behav Neurosci 107:618-626.

8. Giovannini MG, Cerbai F, Bellucci A, Melani C, Grossi C, Bartolozzi C, Nosi D and Casamenti F (2008) Differential activation of mitogen-activated protein kinase signalling pathways in the hippocampus of CRND8 transgenic mouse, a model of Alzheimer's disease. Neuroscience 153: 618-633.

9. Haddad JJ (2004) Mitogen-activated protein kinases and the evolution of Alzheimer's: a revolutionary neurogenetic axis for therapeutic intervention? Prog Neurobiol 73:359-377.

10. Kumaran D, Udayabanu M, Kumar M, Aneja R and Katyal A (2008) Involvement of angiotensin converting enzyme in cerebral hypoperfusion induced anterograde memory impairment and cholinergic dysfunction in rats. Neuroscience 155:626-639.

11. Ni J, Ohta H, Matsumoto K and Watanabe H (1994) Progressive cognitive impairment following chronic cerebral hypoperfusion induced by permanent occlusion of bilateral carotid arteries in rats. Brain Res 653:231-236.

12. Ni JW, Matsumoto K, Li HB, Murakami Y and Watanabe H (1995) Neuronal damage and decrease of central acetylcholine level following permanent occlusion of bilateral common carotid arteries in rat. Brain Res 673:290-296.

13. Nilsson OG, Shapiro ML, Gage FH, Olton DS and Bjorklund A (1987) Spatial learning and memory following fimbria- fornix transection and grafting of fetal septal neurons to the hippocampus. Exp Brain Res 67:195-215.

14. Ohta H, Nishikawa H, Kimura H, Anayama H and Miyamoto M (1997) Chronic cerebral hypoperfusion by permanent internal carotid ligation produces learning impairment without brain damage in rats. Neuroscience 79:1039-1050.

15. Otori T, Katsumata T, Muramatsu H, Kashiwagi F, Katayama Y and Terashi A (2003) Long-term measurement of cerebral blood flow and metabolism in a rat chronic hypoperfusion model. Clin Exp Pharmacol Physiol 30:266-272.

16. Roman GC, Wilkinson DG, Doody RS, Black SE, Salloway SP and Schindler RJ (2005) Donepezil in vascular dementia: combined analysis of two large-scale clinical trials. Dement Geriatr Cogn Disord 20:338-344.

17. Sarti C, Pantoni L, Bartolini L and Inzitari D (2002) Persistent impairment of gait performances and working memory after bilateral common carotid artery occlusion in the adult Wistar rat. Behav Brain Res 136:13-20.

18. Sharp SI, Francis PT, Elliott MS, Kalaria RN, Bajic N, Hortobagyi T and Ballard CG (2009) Choline acetyltransferase activity in vascular dementia and stroke. Dement Geriatr Cogn Disord 28:233-238.

19. Takizawa S, Fukuyama N, Hirabayashi H, Kohara S, Kazahari S, Shinohara Y and Nakazawa H (2003) Quercetin, a natural flavonoid, attenuates vacuolar formation in the optic tract in rat chronic cerebral hypoperfusion model. Brain Res 980:156-160.

20. Tomimoto H, Akiguchi I, Suenaga T, Nishimura M, Wakita H, Nakamura S and Kimura J (1996) Alterations of the bloodbrain barrier and glial cells in white-matter lesions in cerebrovascular and Alzheimer's disease patients. Stroke 27:20692074.

21. Tomimoto H, Ihara M, Wakita H, Ohtani R, Lin JX, Akiguchi I, Kinoshita M and Shibasaki H (2003) Chronic cerebral hypoperfusion induces white matter lesions and loss of oligodendroglia with DNA fragmentation in the rat. Acta Neuropathol 106:527-534.

22. Ueno M, Tomimoto H, Akiguchi I, Wakita $\mathrm{H}$ and Sakamoto H (2002) Blood-brain barrier disruption in white matter lesions in a rat model of chronic cerebral hypoperfusion. J Cereb Blood Flow Metab 22:97-104.

23. Wakita H, Tomimoto H, Akiguchi I and Kimura J (1994) Glial activation and white matter changes in the rat brain induced by chronic cerebral hypoperfusion: an immunohistochemical study. Acta Neuropathol 87:484-492.

24. Wakita H, Tomimoto H, Akiguchi I, Matsuo A, Lin JX, Ihara M and McGeer PL (2002) Axonal damage and demyelination 
in the white matter after chronic cerebral hypoperfusion in the rat. Brain Res 924:63-70.

25. Wang J, Zhang HY and Tang XC (2009) Cholinergic deficiency involved in vascular dementia: possible mechanism and strategy of treatment. Acta Pharmacol Sin 30:879-888.

26. Williams B, Granholm AC and Sambamurti K (2007) Age- dependent loss of NGF signaling in the rat basal forebrain is due to disrupted MAPK activation. Neurosci Lett 413:110114.

27. Williams BJ, Bimonte-Nelson HA and Granholm-Bentley AC (2006) ERK-mediated NGF signaling in the rat septo-hippocampal pathway diminishes with age. Psychopharmacology (Berl) 188:605-618. 